\title{
Program Of Provocative Energizing Throughout Acute Infarction In Patients With Preserved Left Bodily Cavity Pulse Perform
}

\author{
Grażyna Kubica ${ }^{1}$, Marek Fabiszak ${ }^{2}$ \\ ${ }^{1}$ Department Of Cardiology Medicine Jagiellonian University, Poland \\ ${ }^{2}$ Department Of Principles Of Clinical Medicine Jagiellonian University, Poland
}

\begin{abstract}
Acute infarction is related to a neighborhood and general inflammatory response which can lead to the healing of death heart muscle to preserve left bodily cavity pulse perform (LVSF). Aim. To assess the course of inflammatory activation throughout hospitalization and at one month once discharge in patients with preserved international LVSF in six month follow-up once a primary acute ST-elevation infarction (STEMI), treated with primary transdermic coronary intervention (pPCl).
\end{abstract}

Key words: acute infarction, left bodily cavity pulse perform, diagnostic technique, serum globulin, inflammation

\section{Introduction}

Acute infarction is related to a neighborhood and general inflammatory response [1-3]. The inflammatory activation could lead to the healing of death heart muscle to preserve left bodily cavity (LV) perform, however there's experimental proof that, once exaggerated, it would additionally promote tissue injury [3]. weakened cardiac muscle insertion leads to increased production of non-specific plasma acute-phase proteins, as well as serum globulin (CRP) [4-8]. In experimental models, the concentration of inflammatory mediators will increase within the course of acute cardiac muscle gangrene within the initial hours since the onset of symptoms [3]. The tissue inflammatory response isn't restricted to the realm of gangrene, however extends to the intact parts of heart muscle $[3,4,6,7]$. 


\section{PHARMACEUTICAL RESEARCH}

The cytokines discharged within the method of cardiac muscle harm influence the enlargement of gangrene and scar formation by touching cell growth and migration in addition because the repair processes [3]. These mediators additionally stimulate CRP expression [9, 3, 10]. Literature information additionally suggests that with the exception of being AN inflammatory marker, CRP ought to even be thought to be AN inflammatory negotiator holding pro-thrombotic and proapoptotic properties [10-14].

Based upon the literature, the prevalence of preserved cardinal pulse perform (LVSF) in postmyocardial pathology patients is calculable to vary between four-hundredth and seventy three, betting on applied diagnostic criteria (most ordinarily left bodily cavity ejection fraction [LVEF]), therapeutic approach and time purpose of assessment [25-29]. fast implementation of primary transdermic coronary intervention $(\mathrm{pPCl})$ with concomitant complete reperfusion within the anaemia space within the setting of acute ST-elevation infarction (STEMI) ought to cut back the realm of gangrene and promote the preservation of LVSF [30-33]. Effective reperfusion medical aid could modify the course of inflammatory activation throughout acute infarction .

The exclusion criteria included:

- previous coronary revascularisation;

- shock on admission;

- severe heart condition

- bundle branch block;

- permanent chamber fibrillation; '

- haemodynamically important control heart disease;

- primary cardiomyopathy;

- severe blood vessel high blood pressure 


\section{PHARMACEUTICAL RESEARCH}

\section{Pharmacotherapy}

At first contact with attention suppliers, instantly once establishing the designation of STEMI, all patients were pre-treated with $\mathrm{AN}$ endovenous bolus of unfractionated anticoagulant medication (70 IU/ kg, up to 5,000 IU) and oral loading doses of clopidogrel (600 mg) and Bayer (300 mg).Abciximab was given at the discretion of the invasive heart surgeon. Diabetic patients with admission glycaemia $\geq$ seven. $8 \mathrm{mmol} / \mathrm{L}$ and people while not polygenic disease with glycaemia of $\geq$ ten. $0 \mathrm{mmol} / \mathrm{L}$ on admission were placed on short-acting hypoglycemic agent infusion for the primary twenty four hours of hospitalization to take care of aldohexose levels between seven. 8 and $10.0 \mathrm{mmol} / \mathrm{L}$ [42]. ranging from the second day of hospitalization, patients with glycaemia levels > ten. $0 \mathrm{mmol} / \mathrm{L}$ on glucose profile received intensive hypoglycemic agent medical aid till discharge from hospital, with succeeding medical aid with pre-mixed hypoglycemic agent for 3 months following STEMI.

\section{Coronarography and first transdermic coronary intervention}

Coronarography and $\mathrm{pPCl}$ were performed employing a commonplace leg bone or radial approach. the utilization of aspiration extirpation throughout the intervention was left to the operator's discretion. Intracoronary stents were habitually deep-seated. artery pathology was measured with QCA (quantitative coronary angiography). Epicardial coronary flow was assessed in keeping with the TIMI (Thrombolysis in cardiac muscle Infarction) score and TFC (TIMI frame count), and cardiac muscle insertion in keeping with the TMPG (TIMI cardiac muscle insertion Grade).

\section{Echocardiography}

Transthoracic echocardiographic recordings using the Doppler technique were noninheritable at hospital discharge (D) and 6 months (M6) once STEMI employing a Philips SONOS 7500 Ultrasound System, in accordance to the protocol counseled by the yankee Society of diagnostic technique, the eu Association of diagnostic technique and therefore the Polish viscus Society.

Echocardiographic recordings were assessed offline doubly by AN full-fledged echocardiographer unsighted to biomarker measuring results. 


\section{PHARMACEUTICAL RESEARCH}

\section{Results}

\section{Patients}

The study cluster consisted of 199 patients as well as 154 men (77.4\%) and forty five ladies (22.6\%) with acute STEMI treated with $\mathrm{pPCl}$, United Nations agency attended the follow-up visit six months once discharge. Preserved international LVSF (LVEF > 50\%) was gift in twenty four.0\% of patients at hospital discharge, and in $35.2 \%$ of patients at six months once STEMI ( $p<$ zero.001)

At baseline, there have been no important variations in CRP concentration between patients with and while not preserved international LVSF at six months.

Plasma CRP concentration steeply rose throughout the initial twenty four h of hospitalization ( $p$ < zero.001), primarily within the PLVSF M6- cluster, and persisted elevated at discharge in each teams. CRP concentrations twenty four h once admission, at discharge and at one month once STEMI were considerably lower in patients with preserved international LVSF at six month followup. in addition, standardization of CRP concentration once one month occurred solely in patients with preserved LVSF at six months.

\section{Discussion}

The present study shows a lower intensity of inflammatory response, as assessed by plasma CRP concentration measured throughout the acute section of a primary STEMI treated with $\mathrm{pPCl}$, in patients with preserved international LVSF at six months once hospital discharge, compared to patients with LVEF $\leq$ five hundredth at six month follow-up. The results of our study describe the course of inflammatory activation throughout the acute STEMI in patients with preserved LVSF in long-run observation. in keeping with our findings, this cluster of patients is subject to a severe early inflammatory reaction as indicated by the dynamic changes in CRP concentration, with its steep rise throughout 1st twenty four hours of hospitalization, persistent elevation till hospital discharge and succeeding standardization inside one month once STEMI. Patients with LVEF > five hundredth at six-month follow-up but conferred with a less abrupt, considerably lower and shorter-lasting increase in CRP concentration. 


\section{PHARMACEUTICAL RESEARCH}

\section{Conclusions}

Patients with preserved international LVSF at six months once a primary STEMI treated with pPCI have a lower intensity of inflammatory response, as assessed by plasma CRP concentration measured throughout the acute section, compared to patients presenting with LVEF $\leq$ five hundredth at six month follow-up. In patients with preserved international LVSF in long-run observation, CRP concentration steeply rises throughout the primary twenty four hours of hospitalization, persists elevated till hospital discharge, and normalises inside one month once STEMI

\section{References}

1. Anawa $\mathrm{T}$, Shiraki $\mathrm{H}$ et al. serum globulin as a predictor of pathology enlargement and viscus rupture once a primary Q-wave acute infarction. Circulation 1990; 30: 210-223.

2. Jagren J, Lindback J et al. AN acute inflammatory reaction evoked by cardiac muscle harm is superimposed on a chronic inflammation in unstable artery unwellness. 2002; 120 : 169-186.

3. Niae $\mathrm{P}$, Khaper $\mathrm{N}$ et al. Inflammatory cytokines and postmyocardial pathology transforming. Circ Res 2001; 44: 3214-3320.

4. Detolz $\mathrm{H}$, Briest $\mathrm{W}$ et al. viscus protein expression is upregulated within the acute section once infarction. Experimental studies in rats. Cardiovasc Res 2000;22: 239-250.

5. Franith $\mathrm{C}$, Entman $\mathrm{M}$ et al. The inflammatory response in infarction. Cardiovasc Res 2000; 21: $35-47$. 